\title{
PROPUESTA DE DISEÑO DE UN CENTRO CULTURAL EN EL MUNICIPIO DE FLORIDABLANCA
}

Angélica Lucía Castillo Suárez*

Imagen I. Vista oriente fachada ajardinada Fuente: Elaborado por la autora

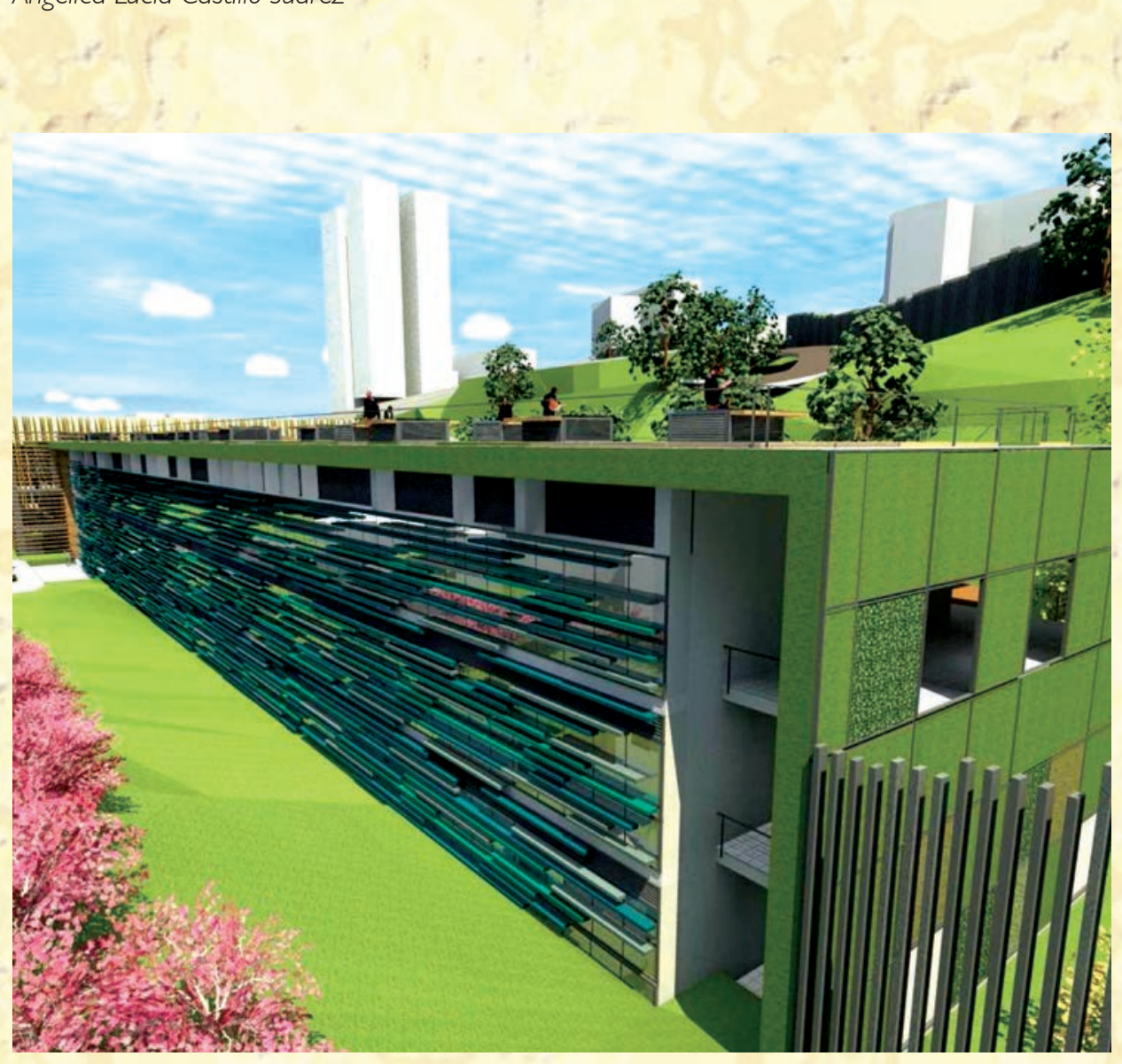

\section{RESUMEN}

La implantación del proyecto fue propuesta para Floridablanca, municipio perteneciente al área metropolitana de Bucaramanga. Su situación estratégica aledaña a la vía de expansión Avenida el Bosque que comunicará la autopista con el anillo vial, favorece en forma notable la fácil y rápida accesibilidad de los usuarios potenciales. La propuesta de implantación del proyecto, obedece al aprovechamiento de un sector del lote con óptimas características topográficas, ya que un gran porcentaje del terreno presenta fuerte pendiente.

\section{PALABRAS CLAVE}

Centro Cultural, Floridablanca, Edificaciones de alta complejidad simbólica. 


\section{PROPOSED DESIGN OF A CULTURAL CENTER IN THE MUNICIPALITY OF FLORIDABLANCA}

Angélica Lucía Castillo Suárez*

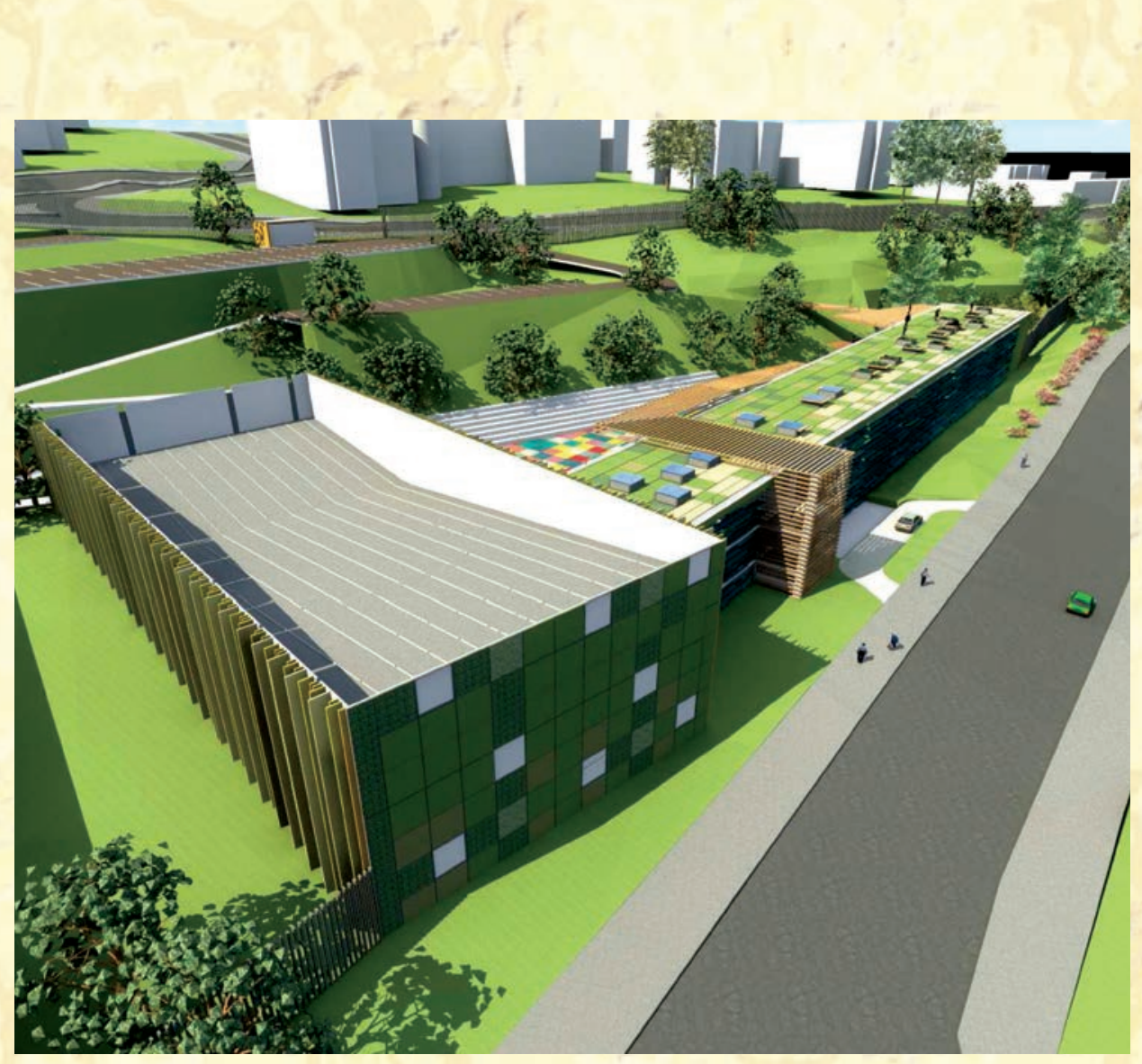

* Ejercicio académico desarrollado en el Taller VIII Edificaciones de alta complejidad simbólica en la Facultad de Arquitectura de la Universidad Santo Tomás, Campus Floridablanca, en diciembre de 2010 , la cual obtuvo una calificación de 4.7

* Docente tutor de facultad arquitecto Saúl Rugeles Quirós, Especialista en Planificación Urbana, Universidad Nacional de Colombia, Sede Bogotá

\begin{abstract}
The project is located in Floridablanca, municipality that belongs to the metropolitan area of Bucaramanga. Its strategic location, adjacent to the expansion way called the Avenue "EI Bosque" that will link the highway with the ring road, dramatically favors potential users' quick and easy accessibility. The proposed implantation of the project, responses to profit an allotment section with excellent topography, since a large percentage of the ground is concentrated in steep hilly terrain.
\end{abstract}

\title{
KEY WORDS
}

Cultural Center, Floridablanca, Buildings of high symbolic complexity

Imagen 2. Vista aérea de la propuesta. Fuente: Elaborado por la autora 


\section{DETERMINANTES CLIMÁTICAS}

La determinante principal en una ciudad de clima cálido húmedo, son los vientos predominantes del norte; de allí que la edificación expone su cara principal a estas corrientes, con el fin de permitir la ventilación cruzada. Al mismo tiempo fueron empleadas protecciones solares, tanto verticales, para disminuir casi en la totalidad los fuertes rayos solares del oriente y occidente, como horizontales, para disminuir la entrada de rayos solares en las fachadas sur y norte. Asimismo, se implementó el uso de fachadas y cubiertas ajardinadas, en las cuales se deciden algunas perforaciones para implementar la iluminación por reflexión. Todos estos mecanismos se desarrollan en la propuesta con el fin de lograr el menor uso de energía mecánica y brindar confort de los usuarios.

Imagen 3. Articulación con el entorno urbano y paisajístico Fuente: Elaborado por la autora

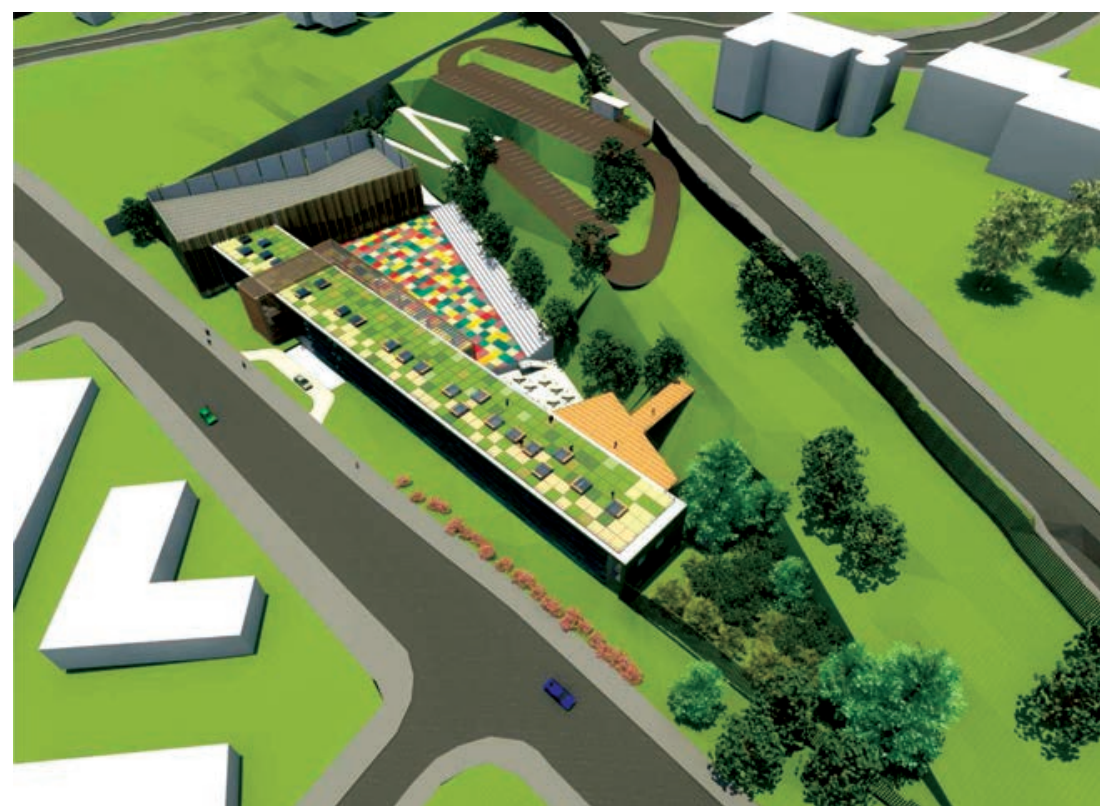

Imagen 4. Cubierta ajardinada transitable Fuente: Elaborado por la autora

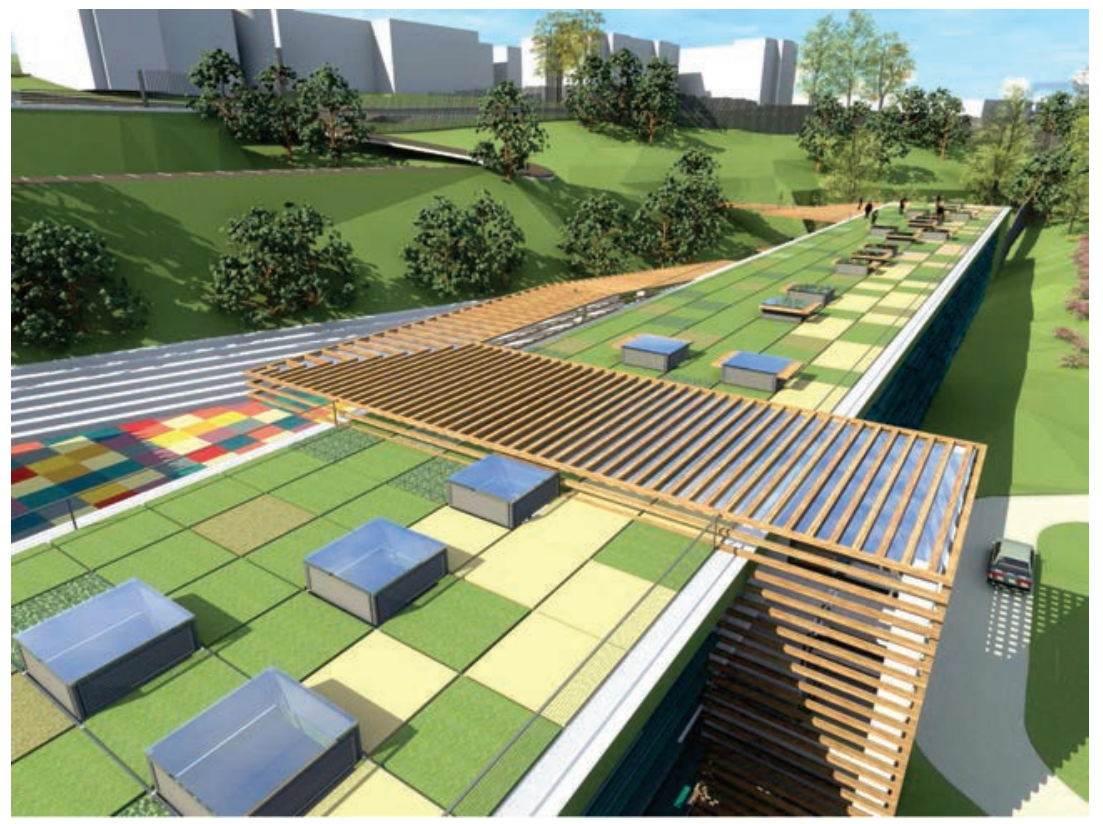




\section{CODIGOS SEMÁNTICOS DEL SIGNIFICADO}

El proyecto ofrece al transeúnte armonía con su entorno, en la cual prima la naturaleza de la colina sobre una arquitectura sutil y sobretodo amable, provocándole a nivel sensorial paz y tranquilidad al caminar, caracterizando así al proyecto como una metáfora de sostenibilidad del paisaje.

\section{COMPONENTES FUNCIONALES}

El proyecto consta de cuatro componentes principales:

- Zona de aulas, tanto teóricas como de práctica de las artes.

- Biblioteca para la consulta especializada de las disciplinas culturales.

- Zona de exposiciones, en donde se encuentra el auditorio con capacidad para 300 espectadores, un salón galería y recorridos con exposición constante.

- Zona con funciones administrativas.

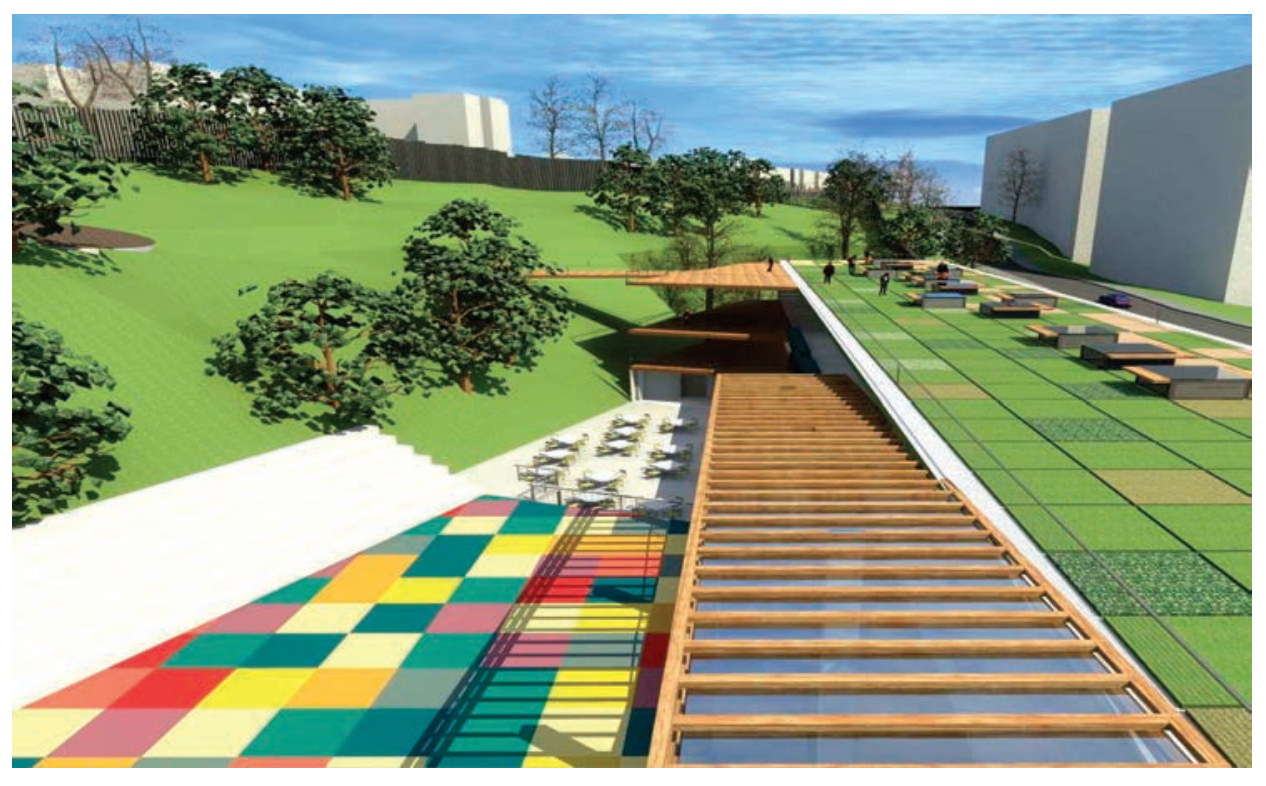

Imagen 5. Vista aérea Occidental Plazoleta de Los Colores. Fuente: Elaborado por la autora 


\section{CENTRO CULTURAL, AUDITORIO Y BIBLIOTECA}

\begin{tabular}{|c|c|}
\hline \multirow{2}{*}{ Locallzación } & Radio de sevicio regional recomendable \\
\hline & Municipio \\
\hline \multirow{2}{*}{ Dotacion } & Poblacion usuaria potencial \\
\hline & Turnos de operación \\
\hline \multirow{3}{*}{$\begin{array}{l}\text { Ubicación respecto a } \\
\text { uso del suelo }\end{array}$} & Recomendable \\
\hline & Condicionado \\
\hline & No recomendable \\
\hline \multirow{3}{*}{$\begin{array}{c}\text { Ubicación en nucleos } \\
\text { de servicio }\end{array}$} & Recomendable \\
\hline & Condicionado \\
\hline & No recomendable \\
\hline \multirow{3}{*}{$\begin{array}{l}\text { Ubicación en } \\
\text { relacion a viabilidad }\end{array}$} & Recomendable \\
\hline & Condicionado \\
\hline & No recomendable \\
\hline
\end{tabular}

$60 \mathrm{~km}$ (1 hora)

Floridablanca / Santander

Personas de 6 años en adelante

8 horas

Comercio. Habitacional. Oficinas y Servicios

N.A

Industrial y No urbano

Subcentro urbano y Corredor urbano

Centro de barrio y Centro urbano

Centro vecinal y Fuera del area urbana

Calle principal, Av. secundaria y Av. principal N.A

Calle peatonal, Calle local y Autopista urbana

\begin{tabular}{|c|c|c|c|c|c|c|}
\hline \multicolumn{4}{|c|}{ Programa Arquilectonico } & \multicolumn{3}{|c|}{ Superficies en $\mathrm{m}^{2}$} \\
\hline AREA & SECTOR & COMPONENTE ARQ. & No. & Area c/u & Construida & Descublerta \\
\hline \multirow{38}{*}{$\begin{array}{l}\text { CENTRO } \\
\text { CULTURAL }\end{array}$} & \multirow[t]{2}{*}{ Acceso } & Hall de acceso principal & 1 & 60 & 60 & \\
\hline & & Recepción & 1 & 13 & 13 & \\
\hline & \multirow[t]{2}{*}{ Aроуо } & Baterias de Baños & 3 & 42,7 & 128,1 & \\
\hline & & Enfermeria & 1 & 30.8 & 30,8 & \\
\hline & \multirow[t]{8}{*}{ Cafeteria } & Zona de mesas & 1 & 360 & 360 & \\
\hline & & Cocina & 1 & 48,5 & 48,5 & \\
\hline & & Depositos - Neveras & 1 & 12 & 12 & \\
\hline & & Mostrador & 1 & 13 & 13 & \\
\hline & & Baños Público & 1 & 20,5 & 20,5 & \\
\hline & & Vestier Empleados & 1 & 5,3 & 5,3 & \\
\hline & & Baño Empleados & 1 & 3 & 3 & \\
\hline & & Deposito Basuras & 1 & 5 & 5 & \\
\hline & \multirow[t]{6}{*}{ Administrativa } & Secretaria & 1 & 13 & 13 & \\
\hline & & Sala de Espera & 1 & 18 & 18 & \\
\hline & & Dirección - Sala de juntas & 1 & 33,5 & 33,5 & \\
\hline & & Oficina Contabilidad & 1 & 20.2 & 20,2 & \\
\hline & & Oficina Programacion Culłural & 1 & 18,5 & 18.5 & \\
\hline & & Sala de Profesores & 1 & 56 & 56 & \\
\hline & \multirow[t]{4}{*}{ Zona Expositiva } & Galeria Exposiciones & 1 & 136 & 136 & \\
\hline & & Deposito & 1 & 19 & 19 & \\
\hline & & Corredores de Exposiciones & - & 136,6 & 136,6 & \\
\hline & & Salón Multiple & 1 & 308 & 308 & \\
\hline & Zona Educativa & Aulas Enseñanza Teorica & 2 & 54,5 & 109 & \\
\hline & Audiovisuales & Salas de Proyeccion & 2 & 54,5 & 109 & \\
\hline & \multirow[t]{3}{*}{ Danza } & Salones de Danza & 2 & 92 & 184 & \\
\hline & & Vestier - Deposito & 2 & 9,5 & 19 & \\
\hline & & Baño & 2 & 4,6 & 9.2 & \\
\hline & \multirow[t]{3}{*}{ Teatro } & Salones de Teatro & 2 & $42, \mathrm{~A}$ & 84,8 & \\
\hline & & Vestier - Deposito & 2 & 9,5 & 19 & \\
\hline & & Baño & 2 & 4,6 & 9,2 & \\
\hline & \multirow[t]{3}{*}{ Artes plasticas } & Salones de Pintura & 2 & $42, A$ & 84,8 & \\
\hline & & Deposito de elementos & 2 & 9,5 & 19 & \\
\hline & & Baño & 2 & 4,6 & 9,2 & \\
\hline & \multirow[t]{5}{*}{ Musica } & Cubiculos de ensayo Individual & 10 & 4,6 & 46 & \\
\hline & & Salas de Ensayo Bandas & 2 & 28 & 56 & \\
\hline & & Aulas Acústicas & 2 & 28 & 56 & \\
\hline & & Zona de Lockers & 1 & 9,3 & 9,3 & \\
\hline & & Subtotal & & & 22815 & 0 \\
\hline
\end{tabular}




\begin{tabular}{|c|c|c|c|c|c|c|}
\hline \multirow[t]{15}{*}{ BIBLIOTECA } & \multirow[t]{4}{*}{ Acceso } & Informacion y Prestamo & 1 & 13 & 13 & \\
\hline & & Sala de reposo & 1 & 21 & 21 & \\
\hline & & Zona de Busqueda & 1 & 6 & 6 & \\
\hline & & Area de Copiado & 2 & 2 & 4 & \\
\hline & \multirow[t]{2}{*}{ Administrativo } & Coordinador Biblioteca & 1 & 20.5 & 20,5 & \\
\hline & & Catalogacion y procesos & 1 & 20.5 & 20,5 & \\
\hline & Medios & Sala Virtual Interactiva & 1 & 97 & 97 & \\
\hline & \multirow{4}{*}{ Colección Abierta } & Exhibicion titulos Nuevos & 1 & 60 & 60 & \\
\hline & & Colección General & 1 & 25 & 25 & \\
\hline & & Colección Infantil & 1 & 15 & 15 & \\
\hline & & Hemeroteca & 1 & 15 & 15 & \\
\hline & \multirow[t]{3}{*}{ Salas de Lectura } & Sala General & 1 & 130 & 130 & \\
\hline & & Sala Infantil & 1 & 55 & 55 & \\
\hline & & Sala Hemeroteca & 1 & 55 & 55 & \\
\hline & & Subtotal & & & $\underline{537}$ & $\underline{0}$ \\
\hline
\end{tabular}

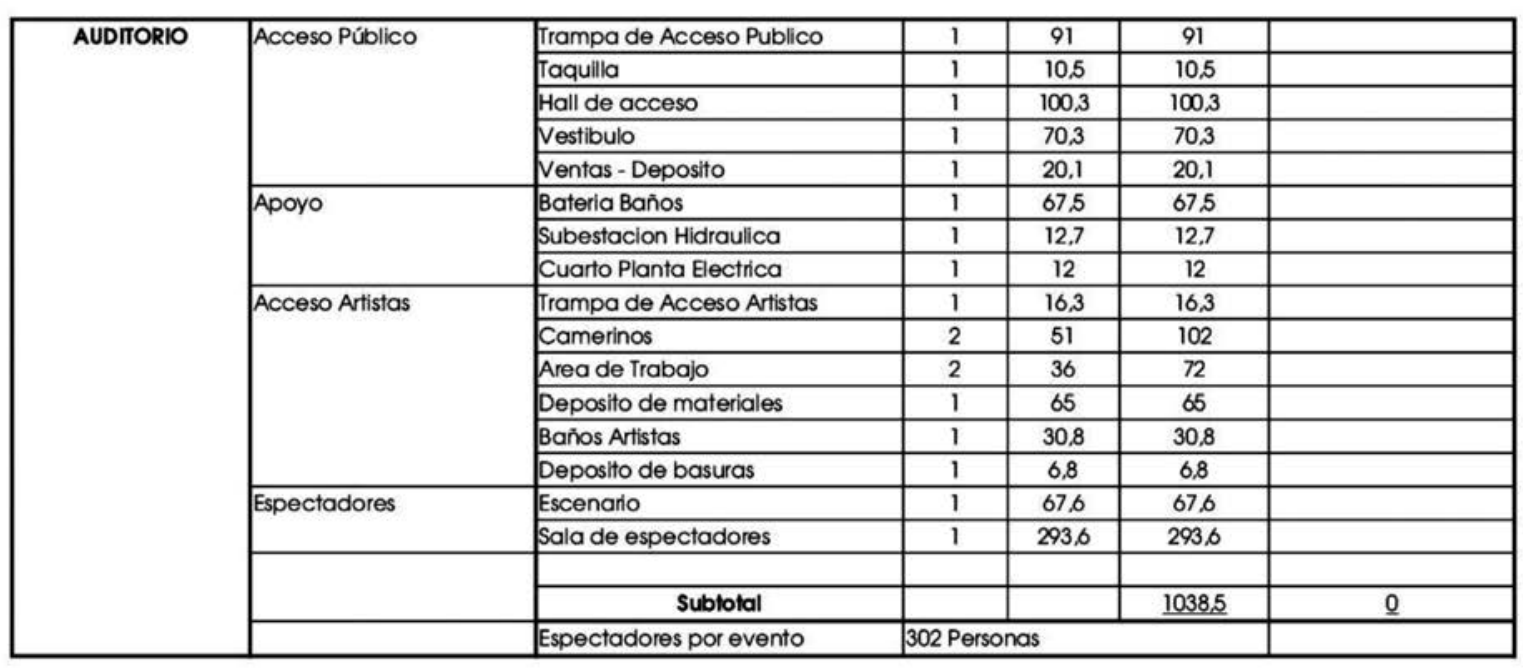

\begin{tabular}{|l|l|l|c|c|c|c|}
\hline Parqueaderos & Parqueaderos & Porteria & 1 & 26,5 & 26,5 & \\
\cline { 3 - 7 } & & Parqueaderos & 72 & & & 2252,3 \\
\hline
\end{tabular}

\begin{tabular}{|l|l|l|l|l|l|l|}
\hline Esparcimiento & Eventos de Encuentro & Plazoleta de los Colores & 1 & 1560 & & 1560 \\
\cline { 3 - 7 } & & Terrazas & - & 418 & 418 & \\
\cline { 3 - 7 } & Cubierta verde Transitable & 1 & 1575 & & 1575 \\
\cline { 2 - 7 } & Areas verdes & - & 4500 & & 4500 \\
\hline
\end{tabular}

\begin{tabular}{|c|c|c|c|c|c|c|}
\hline \multirow[t]{4}{*}{ Circulaciones } & Circulaciones Interiores & Circulaciones & - & $1017 \mathrm{~A}$ & $1017 A$ & \\
\hline & \begin{tabular}{|l|l|l|c|} 
Circulacios Exteriores \\
\end{tabular} & Rampa al Auditorio & 1 & 356,8 & & 356,8 \\
\hline & Distribucion en Plantas & Rampa & 1 & 115 & 115 & \\
\hline & & Escalera & 1 & 33 & 33 & \\
\hline \multirow{5}{*}{\multicolumn{2}{|c|}{ TOTALES PROYECTO $\mathrm{m} 2$}} & \multicolumn{3}{|l|}{ Area Lote } & - & 17881,1 \\
\hline & & \multirow{2}{*}{\multicolumn{3}{|c|}{\begin{tabular}{|l|} 
Area Ocupada \\
Area Total Construida \\
\end{tabular}}} & 31443 & - \\
\hline & & & & & 5466,9 & - \\
\hline & & \multicolumn{3}{|l|}{ Areas Libres Duras } & - & 4169,1 \\
\hline & & \multicolumn{3}{|l|}{ Areas Libres Verdes } & - & 6075 \\
\hline
\end{tabular}

Imagen 6. Programa arquitectónico y cuadro de áreas. Fuente: Elaborado por la autora 

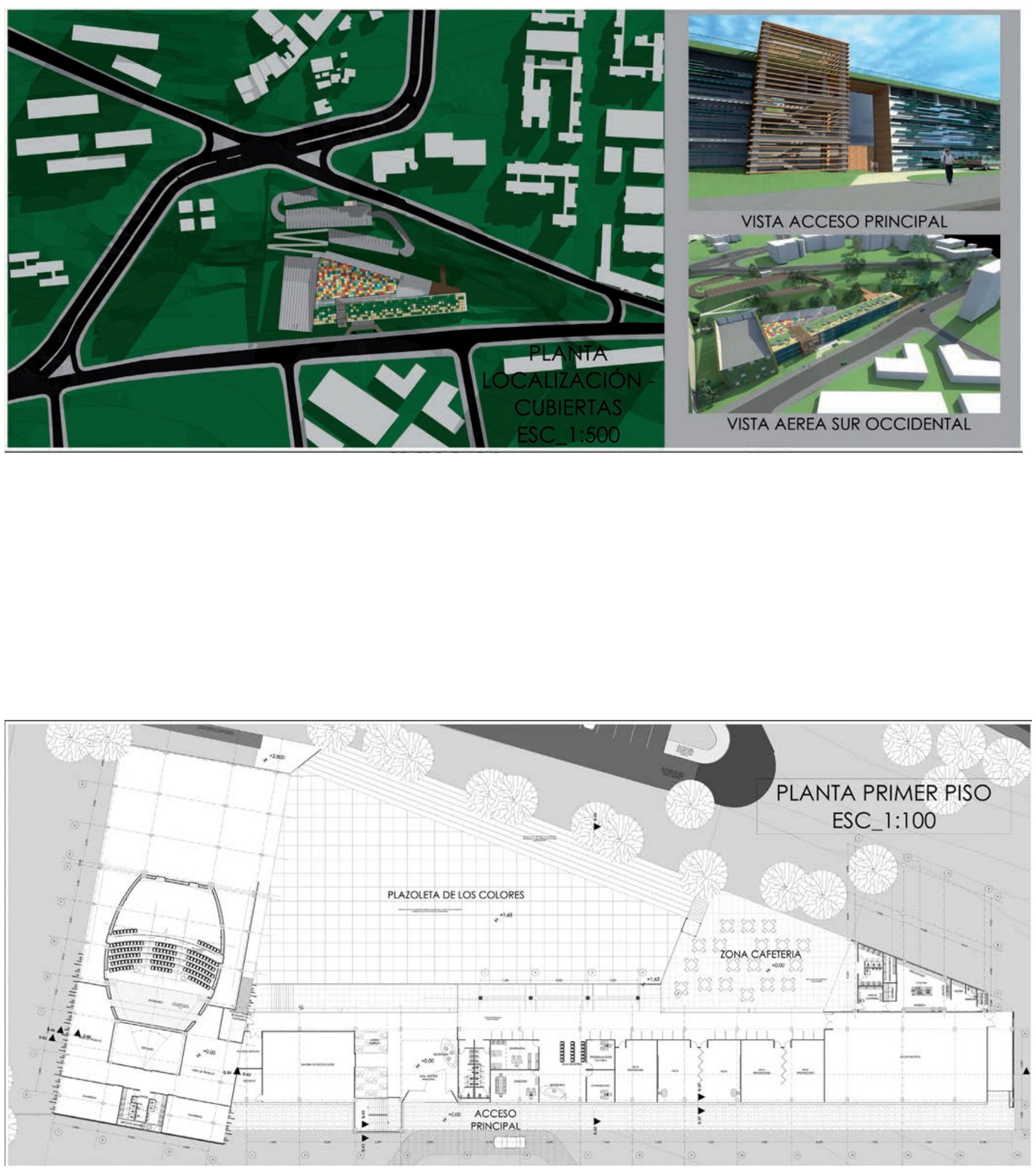

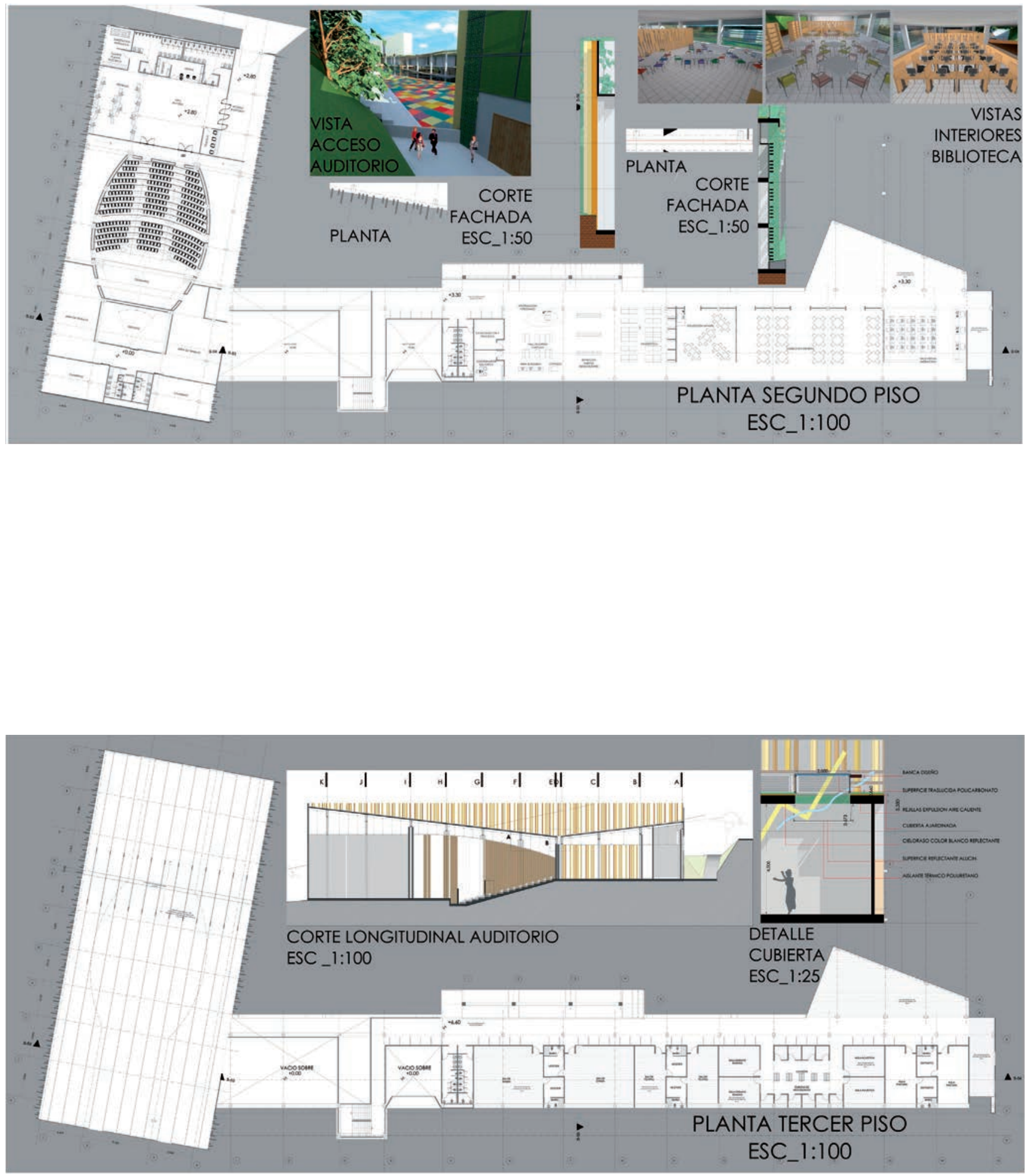

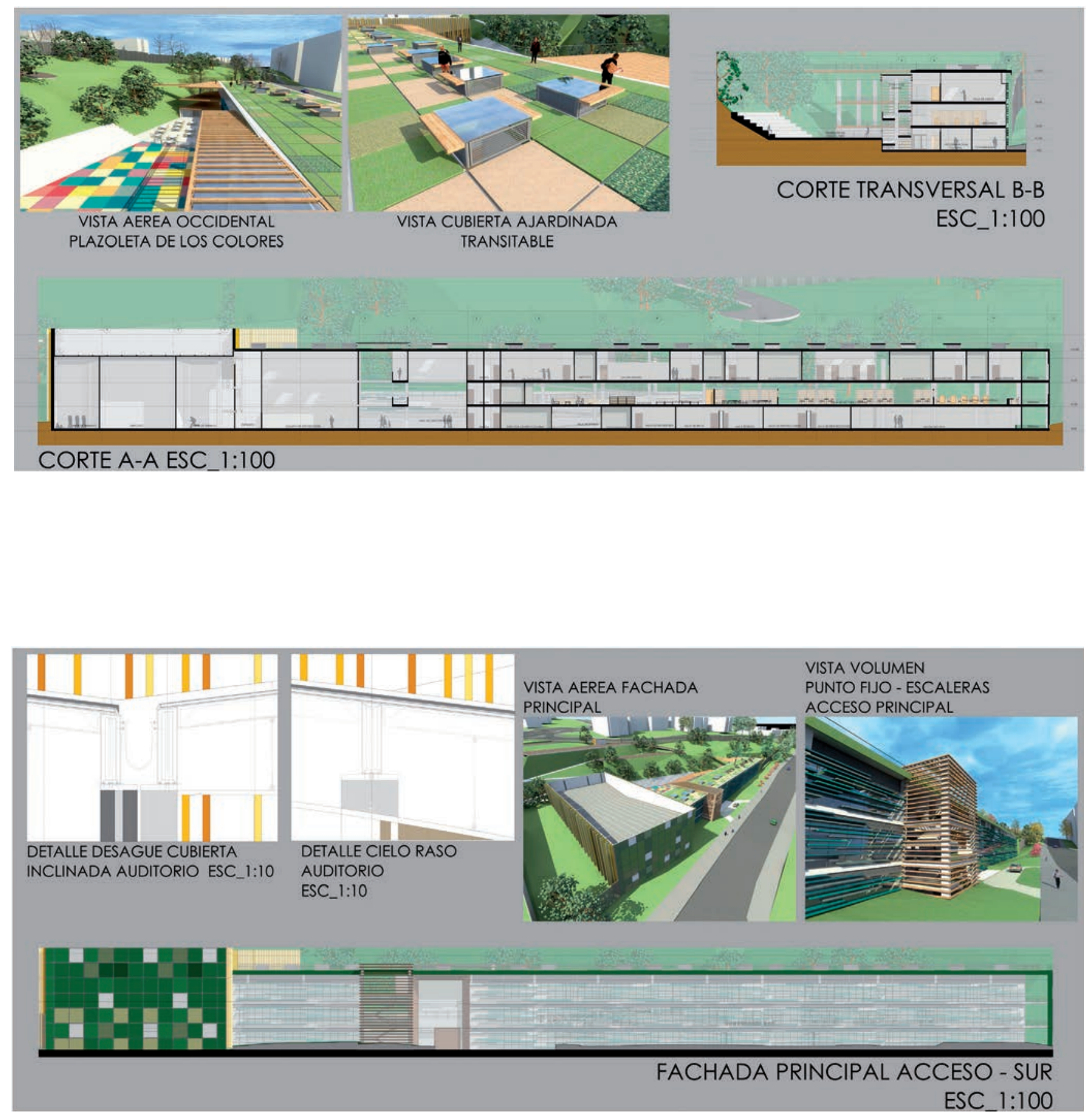


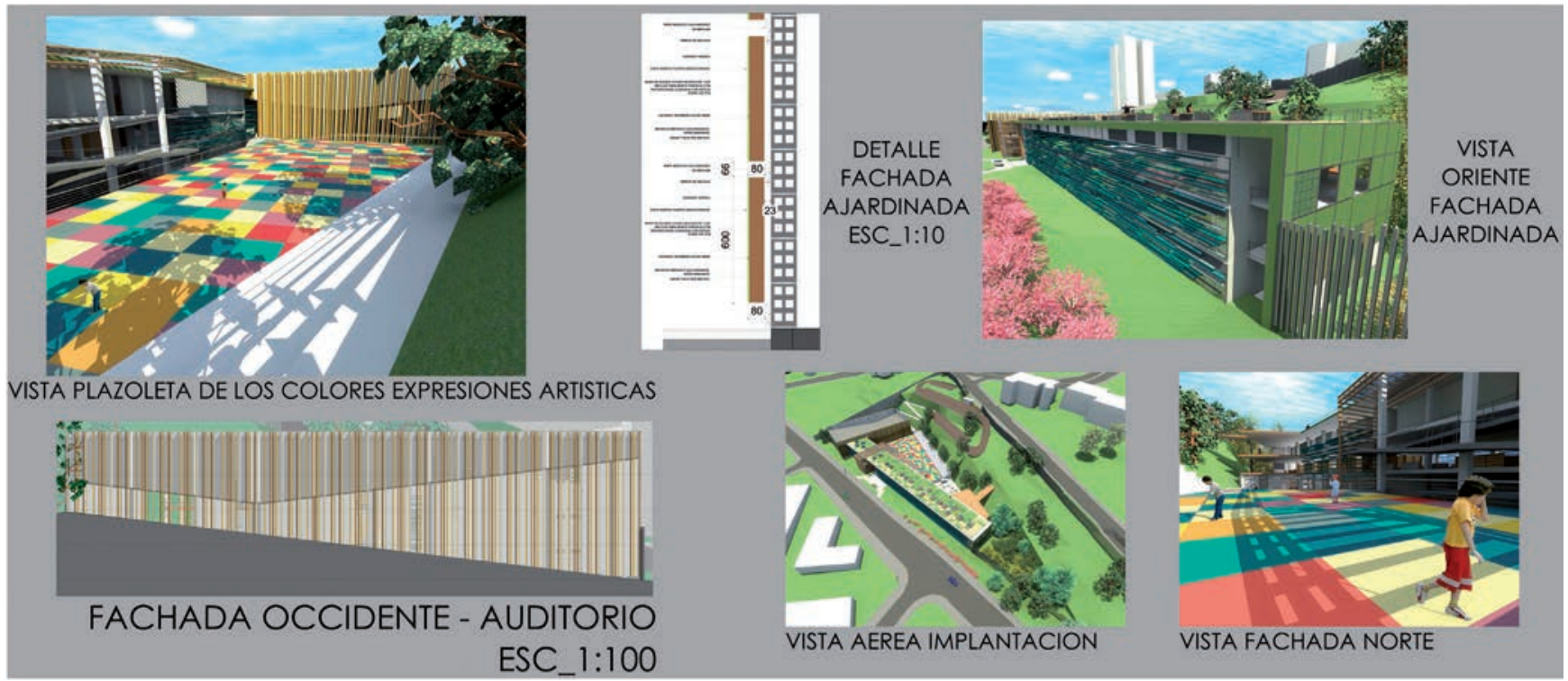

